\title{
Criminologie
}

\section{Pourquoi pas la prévention du crime? Une perspective canadienne}

\section{Lisa Monchalin}

Volume 42, numéro 1, printemps-été 2009

L’intervention à l'aune des données "probantes "

URI : https://id.erudit.org/iderudit/029810ar

DOI : https://doi.org/10.7202/029810ar

Aller au sommaire du numéro

\section{Éditeur(s)}

Les Presses de l'Université de Montréal

ISSN

0316-0041 (imprimé)

1492-1367 (numérique)

Découvrir la revue

Citer cet article

Monchalin, L. (2009). Pourquoi pas la prévention du crime ? Une perspective canadienne. Criminologie, 42(1), 115-142. https://doi.org/10.7202/029810ar
Résumé de l'article

Cet article offre un aperçu des travaux scientifiques portant sur les programmes efficaces de prévention du crime et sur leur mise en oeuvre. Les évaluations scientifiques de projets de prévention du crime qui abordent des facteurs de risque montrent fréquemment qu'ils peuvent réduire la criminalité et que, souvent, ils sont plus efficaces à le faire que les réponses habituelles de la justice pénale. Les organisations intergouvernementales s'entendent sur les étapes-clés qui sont nécessaires pour en arriver à mobiliser les organismes concernés dans la lutte contre ces facteurs de risque. Malgré les recommandations de comités parlementaires et d'un nombre croissant d'experts, la prévention du crime n'est pas encore arrivée à jouer le rôle prépondérant qui pourrait être le sien afin de réduire plus efficacement les taux de criminalité au Canada. Toutefois, la politique récente annoncée par la province de l'Alberta suggère quelques avenues par lesquelles cette résistance pourrait éventuellement être surmontée. 


\section{Pourquoi pas la prévention du crime? Une perspective canadienne}

Lisa Monchalin

Doctorante

Institut pour la prévention du crime, Université d'Ottawa

Lisa.Monchalin@uottawa.ca

RÉSUMÉ - Cet article offre un aperçu des travaux scientifiques portant sur les programmes efficaces de prévention du crime et sur leur mise en œuvre. Les évaluations scientifiques de projets de prévention du crime qui abordent des facteurs de risque montrent fréquemment qu'ils peuvent réduire la criminalité et que, souvent, ils sont plus efficaces à le faire que les réponses habituelles de la justice pénale. Les organisations intergouvernementales s'entendent sur les étapes-clés qui sont nécessaires pour en arriver à mobiliser les organismes concernés dans la lutte contre ces facteurs de risque. Malgré les recommandations de comités parlementaires et d'un nombre croissant d'experts, la prévention du crime n'est pas encore arrivée à jouer le rôle prépondérant qui pourrait être le sien afin de réduire plus efficacement les taux de criminalité au Canada. Toutefois, la politique récente annoncée par la province de l'Alberta suggère quelques avenues par lesquelles cette résistance pourrait éventuellement être surmontée.

MotS-CLÉs - Prévention du crime, données probantes, implantation de programmes, Canada.

\section{Introduction}

Au cours des dernières années, un corpus important de connaissances s'est développé dans le champ de la prévention du crime. De nombreuses études scientifiques (Hahn et al., 1994; Olds et al., 1999; Schweinhart, 2005), ainsi que plusieurs recensions des écrits (Sherman et al., 1997; Sherman et al., 2002; Waller, 2006) ont conclu que les efforts de prévention qui ciblent des facteurs de risque peuvent réduire

Traduit de l'anglais par Denis Lafortune et Sandie Blancher. 
la criminalité1. Au Canada, en 1993, le Comité permanent de la justice et du Solliciteur général, également connu sous le nom de «Comité Horner», a analysé les données qui étaient disponibles à l'époque et fait plusieurs recommandations relatives à la prévention du crime. Il a conclu que, sur une période de cinq ans, au moins $5 \%$ des budgets fédéraux en matière de justice pénale devraient être redirigés vers la prévention du crime et qu'un cadre supérieur devrait être désigné au gouvernement pour aller de l'avant en cette matière (Horner, 1993).

Malgré plusieurs efforts ${ }^{2}$, tel que nous allons le voir, peu de progrès ont été accomplis dans la réalisation du potentiel qu'a la prévention de réduire la criminalité. De plus, en dépit d'une augmentation significative de l'ensemble des données montrant que certaines stratégies préventives sont efficaces, et des appuis qu'ont apportés à ces preuves de prestigieux organismes intergouvernementaux et scientifiques, les gouvernements fédéral, provinciaux et municipaux continuent d'investir massivement dans ces systèmes réactifs traditionnels que sont les services policiers, les tribunaux et les services correctionnels. Dans la lutte contre la criminalité au Canada, la principale réponse reste profondément ancrée dans ces trois piliers traditionnels du système de justice pénale, ainsi que dans les concepts de dissuasion et, de plus en plus, de neutralisation.

La première section de cet article discute des conclusions de diverses évaluations scientifiques qui révèlent qu'une prévention efficace et fondée sur des données probantes en est surtout une qui s'attaque aux facteurs de risque. La deuxième section examine les faits et les concepts qui relèvent d'un nouveau corpus de connaissances portant sur l'implantation de la prévention du crime, en particulier sur le rôle des différents paliers de gouvernement dans la mobilisation d'organismes qui peuvent s'attaquer à un ou plusieurs facteurs de risque qui mènent au crime. Dans la troisième section, la mesure dans laquelle les différents paliers de gouvernement ont concrètement investi dans des stratégies et programmes de prévention efficace pour réduire la criminalité est examinée. Dans la section finale, nous tirons certaines conclusions sur ce qui pourrait favoriser un plus grand investissement dans la prévention efficace du crime.

1. Si ce corpus est bien connu en langue anglaise, il est malheureusement moins diffusé en langue française.

2. Par exemple, la mise en place du Conseil national de prévention du crime (instauré en 1994 à la suite d'une recommandation du Comité permanent de la justice et du Solliciteur général), du Centre international pour la prévention du crime (CIPC; en 1994) et récemment, de l'Institut pour la prévention du crime (IPC). 


\section{Définir la prévention du crime}

Avant d'identifier dans les écrits scientifiques les pratiques considérées comme efficaces, il faut d'abord définir la notion de prévention du crime. En 2002, Cusson a décrit la prévention de la «délinquance» (l'auteur préfère ce terme à celui de crime) comme étant centrée sur l'identification et la réduction subséquente des causes du crime; l'espoir est qu'une intervention préventive en vienne à briser un des maillons rapprochés de la chaîne causale qui aurait abouti au crime si l'enchaînement n'avait été brisé. Plus récemment (Cusson et al., 2007), cet auteur a considéré que la prévention de la délinquance est simplement une façon proactive et non coercitive d'intervenir dans le but de réduire la fréquence et la sévérité des infractions criminelles. Au cours des 20 dernières années, la signification du concept de prévention du crime est devenue beaucoup plus large. Dans ce contexte, la référence à un système de classification emprunté au modèle médical a permis de définir une prévention du crime à trois niveaux: primaire, secondaire et tertiaire.

La prévention primaire désigne les efforts déployés pour ajuster les politiques universelles et les conditions de vie, sur le plan social, éducatif et économique. Elle est dirigée vers la modification des conditions qui, dans l'environnement physique et social, peuvent mener au crime (Brantingham et Faust, 1976) ${ }^{3}$. Les programmes ou les initiatives qui appartiennent à cette catégorie misent sur le lien assez large qui peut être établi entre l'apparition du crime et certains facteurs sociaux ou environnementaux.

La prévention secondaire est une intervention de type «pré-crime» (et tel est l'angle choisi dans cet article). Ciblant des personnes et des groupes à risque d'être incriminés ou victimisés, elle repose sur un dépistage précoce, suivi d'interventions dans la vie des individus ou des groupes considérés à risque d'être impliqués dans un crime. Une fois que les individus, situations, lieux ou opportunités à risque sont identifiés, des interventions conçues pour modifier les facteurs de risque sont mises en œuvre dans l'espoir qu'elles puissent empêcher certaines

3. L'Organisation mondiale de la santé utilise une définition quelque peu différente de la prévention primaire, réservant ce terme aux actions qui visent la prévention avant que ne se produise un crime (Organisation mondiale de la santé [OMS], 2002: 15). Cela dit, la plupart des travaux restent fidèles à l'ancienne définition qui désigne plutôt les interventions qui tendent à se centrer d'une manière indirecte sur le crime (Hastings, 1996: $320)$. 
activités criminelles. La prévention situationnelle du crime est un concept qui décrit une prévention qui se situe, elle aussi, au niveau secondaire (Hastings, 1996: 320). Son but est de modifier la conception et l'organisation de l'environnement physique et social, dans l'intention de rendre les opportunités criminelles plus difficiles à saisir, et ce, en augmentant les probabilités de détection, en réduisant les incitatifs ou en développant le "guardianship».

Comparativement à la prévention primaire et secondaire, la prévention tertiaire se concentre sur les efforts consentis après qu'un crime a été commis. Son but premier porte sur la réduction de la récidive (Brantingham et Faust, 1976). La plupart des activités à ce niveau se concentrent sur la détection, la condamnation, la punition ou le traitement correctionnel des personnes contrevenantes (Hastings, 1996: 320). Cette catégorisation des diverses formes de prévention du crime sera une référence utile dans la section suivante, qui porte sur les pratiques préventives considérées efficaces et basées sur des données probantes.

\section{Prévention efficace du crime}

La plupart des recherches indiquant l'efficacité de la prévention du crime tendent à souligner l'importance de s'attaquer spécifiquement aux facteurs qui induisent des risques de délinquance (Hahn et al., 1994; Olds et al., 1999; Welsh et Farrington, 2002; Burrows, 2003 ; Sherman et al., 2002; Wolfe et al., 2005; Schweinhart, 2005; Farrington et Welsh, 2007; Institut pour la prévention du crime [IPC], 2007a). Plusieurs organisations qui ont l'obligation de répondre de leurs responsabilités publiques, telles que l'Organisation mondiale de la santé (OMS, 2002 et 2004) et le Conseil économique et social des Nations Unies (2002), en sont venues aux mêmes conclusions (Waller, 2006).

En recherche, les succès de la prévention du crime ont pu être corroborés au moyen d'essais randomisés contrôlés et d'autres niveaux de preuves empiriques $^{4}$ (ex. : Burrows, 2003 ; Hahn et al., 1994; Olds et al.,

4. Les exemples de prévention efficace ne sont que quelques-uns de ceux habituellement cités dans la littérature. Dans les faits, de nombreux programmes de prévention du crime se sont montrés inefficaces. Mais plutôt que d'utiliser ces échecs pour conclure que toute forme de prévention du crime est infructueuse, les expériences qui ont échoué permettent d'améliorer l'état des connaissances sur ce qui devrait être/ne devrait pas être préconisé à 
1999; Schweinhart, 2005; Wolfe et al., 2005). Globalement, il en ressort que les projets considérés efficaces s'attaquent aux «facteurs de risque» et, inversement, favorisent l'augmentation de «facteurs de protection». Cette approche préventive a été récemment qualifiée de criminologie «développementale» et/ou de prévention «axée sur la réduction des risques» (Farrington et Welsh, 2007: 94). Depuis les années 1990, elle joue un rôle de plus en plus grand dans le champ de la criminologie. La logique de cette approche est très «directe» dans la mesure où il s'agit: "d'identifier les facteurs-clés engendrant des risques de criminalité et mettre en œuvre des méthodes de prévention conçues expressément pour les neutraliser» (Farrington et Welsh, 2007: 95). Cette approche de la prévention est basée sur un bon nombre de recherches qui ont identifié des facteurs susceptibles de se trouver chez les individus, les familles, les groupes de pairs ou dans les communautés, et pouvant induire des risques de délinquance et de violence (ils se retrouvent par conséquent dans la catégorie de la prévention secondaire).

Une telle façon de concevoir une prévention axée sur la notion de risque a été importée par David Hawkins et Richard Catalano en 1992 (Farrington et Welsh, 2007 : 95) de la santé publique et médicale vers les domaines de la psychologie communautaire et de la criminologie. À titre d'exemple, mentionnons que, dans le modèle médical, si des facteurs de risque tels que le tabagisme ou une alimentation riche en gras sont identifiés et leurs effets vérifiés, alors des mesures préventives conçues pour les réduire devraient logiquement mener à une réduction de résultats indésirables (dans cet exemple, une maladie cardiaque). Une approche axée sur le risque peut être appliquée de façon assez similaire en prévention du crime, afin de réduire ou neutraliser l'impact de facteurs associés à l'agression ou la victimisation. Ce modèle postule, par exemple, que des pratiques parentales inconsistantes/pauvres durant l'enfance prédisposent un individu à présenter plus tard dans sa vie un

l'avenir. Quelques exemples maintes fois cités de programmes de prévention considérés inefficaces, mais néanmoins populaires, ont été mis en place par les milieux policiers: Neighbourhood Watch, DARE, et Scared Straight (Sherman et al., 1997; Sherman et al., 2002; Waller, 2006). Compte tenu de l'espace limité dont nous disposons et du fait que notre intention n'est pas de comparer ce qui fonctionne à ce qui ne fonctionne pas, ces programmes ne sont pas discutés dans cet article. Pour les résultats des évaluations non concluantes, voir: Rosenbaum et al., 1985 et Rosenbaum et al., 1986; Pate et al., 1987; Petrosino et al., 2000; Gottfredson et al., 2002. 
risque accru d'agresser ou d'être victimisé. La lutte contre ce facteur de risque par des mesures appropriées, telles que la formation des parents aux pratiques éducatives ou diverses interventions de soutien, devrait mener à une réduction des taux d'agression et de victimisation. Miser sur d'éventuels facteurs de protection est une autre composante de l'approche préventive axée sur le risque. Un facteur «de protection» en est un qui permet de réduire l'effet potentiellement nuisible d'un facteur de risque (IPC, 2008). De manière générale, les facteurs de protection contribuent à a) la neutralisation des facteurs de risque, b) la diminution de la vulnérabilité à l'agression ou à la victimisation et c) l'accroissement d'une résilience durable. Par exemple, la présence de parents «nourriciers» (nurturing) dans un foyer devrait être considérée comme un facteur de protection pour les enfants.

Cette approche de la prévention est amplement corroborée par un solide corpus de données empiriques. Parmi les projets les plus souvent cités, le Prenatal/Early Infancy Project d'Elmira (New York), le Quantum Opportunities Project et le Fourth $R$ Program témoignent de l'efficacité des efforts de prévention centrés sur le risque. Plusieurs de ces projets ont vu leur efficacité soulignée, notamment par le Centre international de la prévention du crime (CIPC), le Centre pour l'étude et la prévention du crime de l'Université de Colorado et l'Institut pour la prévention du crime (IPC) de l'Université d'Ottawa. Ils sont aussi apparus dans plusieurs recensions exhaustives des programmes efficaces de prévention du crime (Sherman et al., 1997; Greenwood et al., 2001; Sherman et al., 2002; Waller, 2006; IPC, 2007a). Afin d'illustrer ce type d'approche préventive, trois projets seront présentés: un s'adressant aux enfants, l'autre aux adolescents et le dernier aux milieux scolaires.

Le Prenatal/Early Infancy Project d'Elmira (New York) ${ }^{5}$ est un programme de prévention axé sur le risque qui a été maintes fois été reconnu pour son efficacité (Sherman, 1997: 10-15; Greenwood et al., 2001 ; Farrington et Welsh, 2002: 26-30; Waller, 2006: 26-27). Ce projet fut évalué au moyen d'un essai randomisé contrôlé incluant un échantillon de $400^{6}$ jeunes femmes vivant à Elmira, dans les environs de New York. Celles-ci avaient un faible revenu (85\%), étaient céliba-

5. Elmira est une ville du County de Chemung, au sud de l'État de New York.

6. Cinq cents femmes ont été invitées à participer au programme et quatre cents d'entre elles y ont été inscrites (Olds et al., 1999: 53). Les femmes ont été divisées en deux groupes, 200 recevant des visites à domicile et les 200 autres recevant les services habituellement offerts dans la région à ce moment-là (Olds et al., 1999: 53). 
taires ou avaient moins de 19 ans (Olds et al., 1999: 53). L'expérience consistait en un programme de visites à domicile d'une durée de 75 à 90 minutes, sur une base hebdomadaire ou mensuelle, par des infirmières relevant de la santé publique, et ce, auprès de mères à haut risque ${ }^{7}$. Dans le scénario typique, la participation des mères était engagée à la fin de leur premier trimestre de grossesse et elles restaient dans le programme jusqu'à ce que l'enfant ait deux ans. Les infirmières ont ainsi pu conseiller les mères sur la santé et le développement de l'enfant, tout en les aidant à développer des relations de soutien avec leurs familles, leurs amis, et des services sociaux ou sanitaires essentiels (Olds et al., 1999: 49).

Au terme de l'évaluation de ce programme ${ }^{8}$, les analyses ont révélé que les mères qui ont reçu la visite d'infirmières étaient moins susceptibles de maltraiter ou de négliger leur enfant, les cas avérés de mauvais traitements et de négligence étant réduits de $80 \%$ dans ce groupe, en comparaison du groupe contrôle (Karoly et al., 1998: 32; Olds et al., 1999: 44). Ces mères étaient aussi moins enclines à retomber enceintes rapidement et, ayant moins d'enfants, elles pouvaient maintenir une meilleure stabilité professionnelle. Il a aussi été observé que ces mères étaient moins sujettes à l'abus de substances et à l'adoption de comportements criminels que celles du groupe contrôle. En outre, à l'âge de 15 ans, chez les enfants des mères ayant reçu des visites à domicile, on mesurait 56\% moins d'arrestations que chez les enfants du groupe témoin. Ces résultats ont pu être reproduits lorsque des programmes similaires ont été implantés dans la communauté afro-américaine de la ville de Memphis (Tennessee) et la communauté mexico-américaine de Denver (Colorado) (Olds et al., 1999: 46). Une analyse des coûts et bénéfices du projet Elmira a révélé un bénéfice net de 18611 \$ par personne, soit plus de quatre fois l'argent investi (Greenwood et al., 2001: 133).

7. Selon Olds et al. (1999), cela signifie que ces mères étaient célibataires, adolescentes ou pauvres (45).

8. Les résultats du programme ont été mesurés à partir de données tirées d'entretiens, d'observations des pratiques parentales et des conditions de vie à domicile, et de l'examen des dossiers médicaux et sociaux depuis la grossesse et jusqu'à ce que l'enfant ait 15 ans (Olds et al., 1999: 53). Au moment du suivi, 15 ans plus tard, des évaluations furent complétées pour 324 participantes. Cet échantillon représente $81 \%$ de la cohorte originale et $87 \%$ des familles n'ont connu aucune mortalité fotale, maternelle ni infantile (Olds et al., 1999: 54).

9. À l'âge de 15 ans. 
Le Quantum Opportunities Project ${ }^{10}$ est un autre exemple communément cité dans lequel une approche de prévention axée sur le risque a été retenue. Ce programme a focalisé ses efforts sur les adolescents de milieux défavorisés en leur fournissant un programme «parascolaire» de type développemental ${ }^{11}$. Le programme était structuré autour d'activités éducatives comprenant 250 heures d'éducation, 250 heures d'activités de «développement» et 250 heures de «services». Il était offert, de la $9^{\mathrm{e}}$ année jusqu'à la fin des études secondaires, aux adolescents fréquentant l'école, ainsi qu'à ceux de la même tranche d'âge qui avaient décroché des études ou quitté leur école de quartier (Hahn et al., 1994 : 6). Les activités éducatives incluaient du tutorat, une formation en informatique, une formation aux compétences familiales et aux habiletés nécessaires à la vie autonome, ainsi que des services d'orientation tels que la planification d'études supérieures ou la recherche d'un emploi à la fin des études (Hahn et al., 1994; Greenwood et al., 1996). Pour leur participation au programme, les adolescents recevaient une légère compensation financière. Par ailleurs, pour chaque somme qu'ils gagnaient au moyen d'un emploi, le programme investissait un montant équivalent dans le Fonds du collège (Hahn et al., 1994; Greenwood et al., 1996). Une évaluation de ce programme a révélé que les adolescents participants étaient plus susceptibles de terminer leurs études secondaires, d'entreprendre des études postsecondaires et de recevoir un prix ou une récompense scolaire. Ils étaient aussi moins susceptibles d'être exclus de leur école et les jeunes filles moins à risque de tomber enceintes comparativement aux jeunes non participantes (Hahn et al., $1994: 15)^{12}$. L'analyse des coûts-bénéfices de cette expérience a révélé que pour chaque dollar investi dans le programme, 3,68 \$ de bénéfices ont ensuite été récupérés.

10. C'était un projet de démonstration implanté dans cinq communautés étatsuniennes différentes, à partir de septembre 1989 (Hahn et al., 1994: 6).

11. Il s'agit d'étudiants d'écoles secondaires, ou ayant l'âge d'être inscrits dans une école secondaire, qui ont été sélectionnés dans une cohorte qui regroupaient des familles bénéficiaires de l'aide sociale. Chaque site a recruté 50 adolescents provenant de milieux défavorisés. Par la suite, 25 d'entre eux ont été assignés à un groupe qui participait au programme en cours de développement et les 25 autres à un groupe témoin (aucun programme; Hahn et al., 1994: 6).

12. Ceci a été montré en comparant le groupe exposé au programme et le groupe témoin, tout au long du programme. Dès septembre 1989 (avant que le programme ne soit commencé), des questionnaires ont été remis aux participants des deux groupes. Ces questionnaires ont de nouveau été remis aux deux groupes une fois le programme commencé, d'abord à l'automne 1990, puis en 1991 et de nouveau en 1992 (Hahn et al., 1994: 7). 
Un troisième exemple de pratique efficace de prévention du crime se trouve dans The Fourth $R$ ( $R$ signifiant "Relationship»), une initiative ayant d'autant plus de pertinence qu'elle est canadienne. Conçu à London (Ontario), ce programme s'attaque aux facteurs de risque associés à la délinquance, la violence domestique ou l'intimidation. Il est présentement mis en œuvre dans certaines écoles sélectionnées à travers le Canada (Wolfe et al., 2005). Il est basé sur un curriculum d'apprentissage d'habiletés réparties en 21 séances et dispensées dans les classes de la $7^{\mathrm{e}}$ à la $10^{\mathrm{e}}$ année par des professeurs ayant les qualifications nécessaires. Les séances valorisent des relations saines. D’autre part, elles ciblent les abus, la violence ${ }^{13}$, les comportements sexuels à risque et l'abus de substances. Ce projet est toujours en cours d'évaluation, mais les premiers résultats d'essais randomisés contrôlés incluant plus de 1500 étudiants de London (Ontario) sont prometteurs et révèlent une baisse significative des comportements agressifs des garçons envers leurs pairs (Wolfe et al., 2005 ; Crooks et al., 2008).

Les programmes mentionnés ci-dessus ne sont que trois exemples tirés des données de recherche de plus en plus nombreuses qui suggèrent que les stratégies de prévention axées sur le risque fonctionnent, et qu'elles le font d'une façon plus efficace et efficiente que les mesures traditionnelles de contrôle du crime, à savoir la police, le système correctionnel et le système de justice pénale (Sherman et al., 1997; Greenwood et al., 2001; Sherman et al., 2002; Waller, 2006). Or, malgré l'existence d'un tel corpus de données, et au lieu de chercher à mettre en œuvre une prévention du crime fondée sur des données probantes, le Canada continue d'investir la plupart de ses ressources humaines et financières dans des formes traditionnelles de réponse au crime et à la victimisation ${ }^{14}$. La prochaine section explore le consensus régnant entre les experts et les organisations publiquement responsables quant à la meilleure manière de mettre en œuvre des programmes efficaces de prévention axés sur des facteurs de risque.

13. Telles l'intimidation, la violence conjugale, la violence entre pairs et la violence de groupe.

14. Comme il sera mentionné et discuté plus tard dans la section «L'évolution de la prévention du crime au Canada». 


\section{La mise en œuvre d'une prévention efficace : données probantes et concepts}

Au Canada, malgré les données probantes accumulées jusqu'ici (et dont nous avons discuté précédemment), une prévention efficace du crime n'a pas encore été implantée à grande échelle. Même si certains programmes gagnent du terrain ${ }^{15}$, globalement leur implantation systématique reste minimale.

Dans ce domaine, plusieurs personnes et organismes restent aux prises avec le défi qui consiste à savoir comment traduire en actions tangibles les connaissances acquises sur «ce qui fonctionne» en matière de prévention du crime. En réponse à cette question difficile, diverses lignes directrices relatives à la manière d'implanter la prévention du crime dans les politiques publiques ont été publiées (Conseil national de prévention du crime, 1996; Waller et al., 1999; Linden, 2000; Shaw, 2001; Conseil économique et social des Nations Unies, 2002; OMS, 2004; Groupe de travail national sur la prévention de la criminalité, 2007). Par ailleurs, diverses organisations nationales et internationales comme l'Organisation mondiale de la santé, les Nations Unies et la Fédération canadienne des municipalités en sont parvenues à un consensus sur la meilleure manière d'implanter une prévention efficace et efficiente.

En 1999, Sansfaçon et Welsh ont analysé une série de recommandations émises par des maires, de même que des avis d'experts ou propositions plus pragmatiques faites par des organismes tels que National Crime Prevention Council des États-Unis et Crime Concern en Angleterre et au Pays de Galles. Selon eux, la plupart des travaux qui décrivent les éléments nécessaires à la mise en œuvre d'une prévention efficace du crime regroupent des thèmes communs. Ces thèmes seront présentés dans les lignes suivantes:

Centres de responsabilité permanents. La recherche tend à soutenir la nécessité de mettre sur pied des centres de responsabilités aux paliers national, provinciaux et municipaux. Ces centres recevraient un financement soutenu (Conseil national de prévention du crime, 1996) et dépendraient du leadership d'un organisme central. Par exemple, dans

15. Le programme Fourth $R$ est actuellement implanté dans plus de 350 écoles en Ontario. Il est adapté et implanté dans six autres provinces canadiennes (Crooks et al., 2008: 111). 
le cas d'un Centre national, on pourrait concevoir qu'une personne supervise l'ensemble de la stratégie canadienne de prévention du crime. Dans le cas des municipalités, cette personne pourrait être quelqu'un exerçant déjà un leadership régional, tel qu'un maire (Waller et al., 1999: 66; Linden, 2000: 3; Waller, 2006).

Mobilisation et coordination de différents secteurs et partenaires. Afin de pouvoir travailler avec les autres, chacun des centres de responsabilité locaux aurait besoin d'un effort de coordination intersectorielle s'exerçant à l'échelle de la municipalité. Cela impliquerait une coordination et des partenariats, aussi bien entre ceux «travaillant dans un ministère et dans un autre», qu'entre «les décideurs, organismes communautaires, organismes non gouvernementaux, secteurs des affaires et citoyens» (Conseil économique et social des Nations Unies, 2002).

Diagnostic de sécurité. Chaque municipalité pourrait exiger un audit sur la sécurité dans sa région et ce, en utilisant des données de Statistiques Canada, des enquêtes autorévélées ou des données recueillies dans les salles d'urgence. Selon l'OMS (2004), ces données sont essentielles car elles dressent un portrait plus précis du problème et permettent de diriger les efforts de prévention vers les secteurs et les groupes qui semblent en avoir le plus besoin.

Élaboration d'un plan d'action. Chaque municipalité aurait alors besoin de développer un plan d'action conçu à partir des problèmes et des lacunes identifiés à l'aide des données statistiques et à travers l'analyse des problèmes criminels spécifiques à certaines municipalités ou régions.

Implantation d'un plan d'action. Chaque municipalité, par l'entremise d'un travail de coordination entre les secteurs et les individus, aurait besoin d'établir ses cibles spécifiques à la lumière des données probantes sur «ce qui fonctionne».

Monitorage et évaluation du plan d'action. De bonnes pratiques requièrent une évaluation continue, afin de déterminer si des modifications devraient être apportées aux différents projets ou initiatives, et une évaluation d'impact afin d'évaluer si la stratégie a produit les effets désirés.

La figure ci-dessous illustre les interactions entre les différentes composantes de la stratégie: 
F I G U RE 1

L'implantation efficace des initiatives de prévention du crime

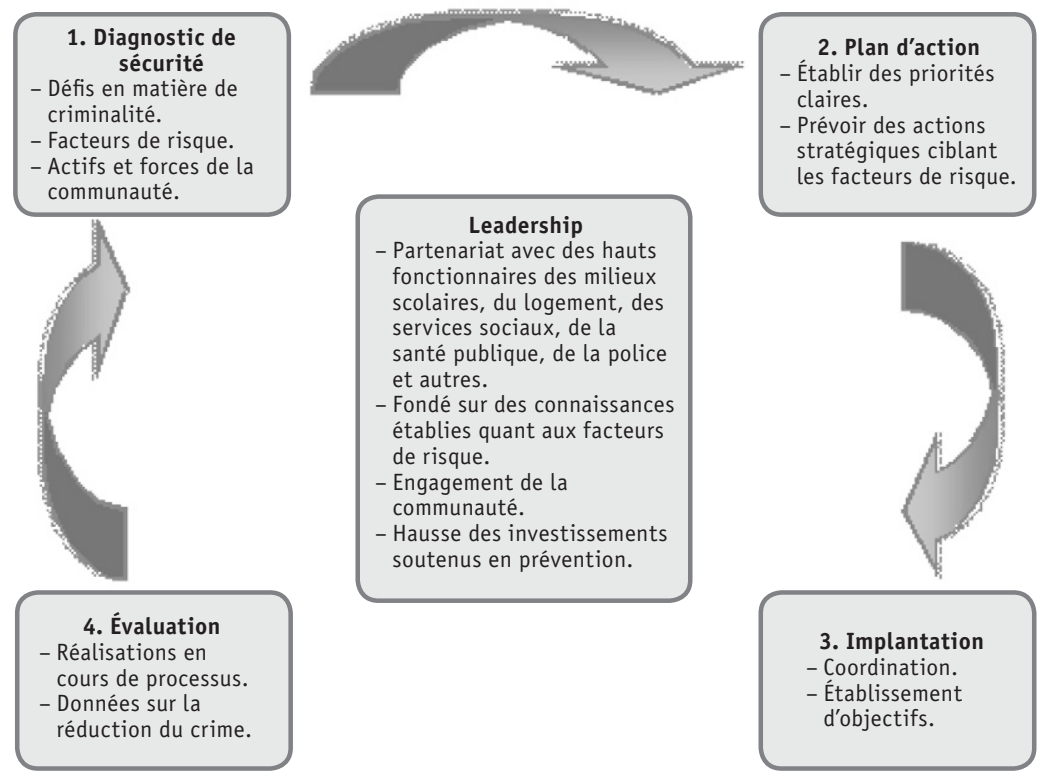

Sources: Waller (2006) et Waller et al. (1999).

Selon Shaw (2001), Waller (2006) et Waller et al. (1999), des dirigeants municipaux élus en France, durant les années 1980, sont à l'origine du modèle d'implantation efficace des initiatives de prévention du crime. En effet, une des premières incarnations de cette façon de faire a pu être observée dans l'Hexagone en 1982, lors des travaux de la Commission des maires sur la sécurité présidée par Gilbert Bonnemaison ${ }^{16}$ (Shaw, 2001: 9). Depuis, le modèle s'est développé sur la base de diverses recommandations émanant de maires, d'experts et d'organismes nationaux (voir Waller, 2006: 116) ${ }^{17}$.

16. À l'époque, M. Bonnemaison, maire d'Épinay-sur-Seine (en banlieue parisienne), fut l'un des premiers à défendre un tel modèle d'implantation multi-organismes.

17. Dans l'ensemble, la recherche scientifique qui porte sur la mise en œuvre de ce modèle reste assez peu concluante. Par conséquent, jusqu'ici, elle n'a donné lieu qu'à certaines applications telles que le «modèle logique». Un modèle logique expose la structure d'un programme. Il établit les liens entre les ressources et les résultats attendus en présentant dans une séquence logique les activités, les réalisations et les résultats pertinents de l'ensemble de la stratégie du programme. 
En somme, entre de nombreux experts, il existe un consensus quant à la meilleure façon d'implanter des programmes de prévention. Cette approche implique que différents paliers de gouvernement puissent mobiliser des organismes gouvernementaux, eux-mêmes luttant de manière systématique contre des facteurs de risque. Elle suppose qu'on commence par diagnostiquer les lacunes, puis ensuite dresser un plan d'action pour les combler, fournir un effort rigoureux pour implanter un programme et, finalement, mettre en place une évaluation.

Au Canada, peu d'exemples de prévention ont fait l'objet d'une implantation efficace de telle sorte que, dans ce pays, les partisans de la prévention du crime continuent de faire face à un bon nombre d'obstacles au moment de transposer en actions concrètes les données probantes sur la prévention. Un état de la situation passée et présente au Canada, quant aux efforts de prévention du crime, sera maintenant présenté.

\section{L'évolution de la prévention du crime au Canada}

Au Canada, bien que la question de la prévention du crime ait été posée aussitôt qu'en $1949^{18}$, et qu'une conférence nationale à ce sujet ait été tenue à l'Université de Toronto en 1945, le champ de la prévention du crime a été négligé pendant plusieurs années. En effet, les politiques publiques sont restées centrées sur les problèmes relatifs aux prisons et au droit pénal, plutôt que de s'orienter vers des stratégies de prévention du crime (Birkbeck, 2005).

Ce n'est que dans les années 1980 que la prévention du crime a véritablement commencé à être plus reconnue au Canada. Un moment décisif dénotant ce mouvement est survenu en 1984 lorsque Irwin Waller et Richard Weiler ont préparé pour le Conseil canadien de développement social le rapport intitulé La prévention du crime par le développement social. Ce rapport a marqué un tournant dans l'histoire de la prévention du crime au Canada, puisqu'il a aidé le gouvernement canadien à finalement admettre et prendre en considération l'importance du champ de la «prévention du crime». Ce document, de même

18. Selon Birkbeck (2005), l'expression «prévention du crime» est d'abord parue dans une série d'articles proposés par la Gendarmerie royale du Canada. Ces articles ont été regroupés dans un ouvrage couvrant 20 sujets différents (Royal Canadian Mounted Police, 1949). Dans un chapitre consacré à la «prévention du crime», un auteur anonyme tentait d'examiner le sujet, sous l'angle de la politique et de la police (Birkbeck, 2005: 325). 
qu'un rapport d'Irwin Waller (1989; voir Begin, 1992) destiné au gouvernement canadien ${ }^{19}$, a été repris presque mot pour mot dans un texte préparé pour le Parlement canadien en 1992 (Birkbeck, 2005 : 331). Ce sont ces mouvements dans le champ de la prévention du crime qui ont inspiré le Comité Horner et l'ont incité à publier en 1993 Prévention du crime au Canada: vers une stratégie nationale (Horner, 1993), un texte que nous appellerons désormais «rapport du Comité Horner».

Le rapport du Comité Horner, adoptant la plupart des orientations suggérées par Waller et Weiler, soulignait l'importance d'une prévention du crime qui passerait par le développement social. Il lançait un appel à la création d'un conseil national de prévention du crime (centre de responsabilité) au palier fédéral, conseil qui travaillerait en partenariat avec les provinces, les territoires et les municipalités, et serait supervisé par un cadre supérieur ou un haut fonctionnaire. Le rapport faisait aussi écho à la recommandation de Waller selon laquelle, pour une première période de cinq ans, $1 \%$ du budget fédéral actuel destiné à la police, aux tribunaux et aux services correctionnels devrait être alloué aux efforts de prévention du crime, cette part atteignant $5 \%$ au bout de cinq ans.

Le rapport du Comité Horner a eu un impact notable sur la reconnaissance de la prévention du crime au Canada. C'est ainsi qu'en 1994, le gouvernement fédéral a lancé la Phase I de la Stratégie nationale pour la prévention du crime et la sécurité publique. La première phase de la Stratégie nationale était axée principalement sur les enfants, les familles et les communautés. Elle a permis de souligner l'importance de la mobilisation communautaire et du renforcement des capacités de la collectivité. Après l'achèvement de la Phase I en 1997, le gouvernement a lancé la Phase II qui prévoyait la création du Centre national de prévention du crime (CNPC). Aujourd'hui, la mission principale du CNPC est la mise en application de la Stratégie nationale pour la prévention du crime au Canada. Dans le Plan d'action visant à diminuer efficacement la criminalité (Sécurité publique Canada, 2008), le CNPC réaffirme sa mission et souligne l'importance de faire preuve de leadership quant aux moyens de s'attaquer aux facteurs de risque connus chez les populations les plus vulnérables et dans les milieux à risque

19. Il s'agit d'une recension complète des stratégies internationales de la prévention du crime, qui, ultimement, a souligné le besoin de créer un conseil national de prévention du crime. 
élevé. L'accent mis sur les facteurs de risque a annoncé un changement dans la stratégie du CNPC, qui est devenue plus axée sur les principes de la prévention efficace du crime.

Cependant, le CNPC manque toujours d'un centre de responsabilité et d'un chef de file, deux éléments essentiels du modèle d'implantation efficace présenté à la figure 1 et des recommandations formulées par le Comité Horner ${ }^{20}$. Il compte encore beaucoup sur une approche allant du bas vers le haut («bottom-up»), où la responsabilité de soumettre des projets de financement de prévention du crime repose entièrement sur les municipalités ou les communautés. Le mandat du CNPC indique que les financements prioritaires iront aux communautés qui sauront faire la démonstration d'une stratégie comportant le renforcement de leurs capacités et des projets centrés sur des personnes ou des groupes qui présentent des facteurs de risque multiples. En regard du Fonds de lutte contre les activités des gangs de jeunes, il est spécifié que le financement sera accordé «à des projets où des organismes communautaires et des municipalités ont déjà collaboré pour évaluer les besoins et ont amorcé une intervention coordonnée et concertée à l'égard du phénomène des gangs dans leur milieu» (Centre national de prévention du crime, 2008).

Le CNPC existe présentement en tant qu'organisme de financement dont la principale fonction est de donner de l'argent à certains projets de prévention du crime. Le Canada n'a pas de plan d'action ni de centre de responsabilité qui prenne en charge le mandat de la prévention du crime sur le plan national. Or, comme on l'a décrit dans la section précédente, un plan d'action et un centre de responsabilité sont des composantes-clés d'une implantation efficace. À l'heure actuelle, d'une manière comparable à ce qui se fait aux États-Unis, les communautés sont interpellées et on attend d'elles qu'elles s'organisent, remplissent des formulaires pour obtenir des subventions et satisfassent à certaines conditions particulières. Le fait d'être axé sur le financement de projets dans des communautés à risque est certes un pas dans la bonne direction, toutefois le

20. Voir dans Horner (1993) la recommandation 1, qui indique que «le gouvernement fédéral, de concert avec les provinces, les territoires et les municipalités, assume un rôle national de premier plan pour prévenir le crime et énonce une politique nationale de prévention de la criminalité» (22); recommandation 2, qui appuie «l'établissement d'un conseil national de prévention du crime» (23); et recommandation 4, qui indique qu'un «cadre supérieur» devrait être désigné pour «énoncer une politique et établir un programme de prévention de la criminalité» (25). 
fardeau de la preuve revient aux communautés qui doivent approcher le CNPC de leur propre initiative, une tâche qui pourrait être difficile dans certains milieux plus désorganisés et défavorisés.

Cependant, quelques communautés au Canada se sont organisées et ont su créer des groupes de travail et/ou des conseils municipaux sur la prévention du crime, en ayant recours à un leadership central et au modèle local d'implantation décrit dans une section antérieure (un de ces exemples étant «Prévention du crime Ottawa»). À ce jour, de tels groupes de travail/conseils municipaux de prévention du crime ont été créés dans quatre villes canadiennes, à savoir Québec, Waterloo, Toronto et Ottawa.

En 1992, Québec était une des premières villes canadiennes à mettre en place un groupe de travail (ou «table ronde») sur la prévention de la criminalité. Des fonctionnaires provenant de plusieurs secteurs (municipalités, communautés, écoles, universités, corps policiers, syndicats de police et agences gouvernementales) l'ont constitué. Cette table ronde a permis d'en arriver à un consensus entre différents ministères, associations et experts, et à produire 25 recommandations qui ont été publiées en 1993 dans un rapport intitulé Pour un Québec plus sécuritaire: partenaires en prévention. Rapport de la Table ronde sur la prévention de la criminalité (Table ronde sur la prévention de la criminalité, 1993). Plusieurs de ces recommandations étaient centrées sur l'implantation de programmes proactifs, fondés sur des données probantes et visant en priorité les jeunes les plus défavorisés, dans leur famille, leur école et leur quartier (19-20). Le rapport préconisait un leadership partagé entre les individus, les organisations de bénévoles, le milieu des affaires, les corps de police et les autres agences sociales, tous travaillant ensemble pour mettre en œuvre des programmes efficaces de prévention du crime.

En 2001, Québec a adopté une politique afin de mettre en place la plupart des recommandations de la table ronde de 1993. Cela incluait des stratégies préventives locales mises en place par les municipalités sur la base de partenariats, ainsi que des stratégies de recherche, de développement et de formation en prévention du crime. Toutefois, cette politique n'a pas été accompagnée par le type de financement qu'avait recommandé la table ronde en 1993. Depuis la création du groupe de travail de Québec, trois autres municipalités ont créé des conseils de prévention du crime: la région de Watterloo en 1993, Toronto en 1999 et Ottawa en 2005. Dans chacune de ces villes se tiennent depuis des 
réunions régulières portant sur des efforts de prévention du crime. Chacune de ces villes a su initier des projets locaux de prévention du crime.

Malgré leurs bases solides, les mouvements locaux de prévention du crime continuent d'avoir relativement peu d'influence sur les pratiques gouvernementales actuelles et ils accomplissent assez peu lorsqu'on les compare à ces mesures de justice mieux établies que sont la police, les services correctionnels ou les tribunaux. Au Canada, les réponses traditionnelles de type réactif continuent de dominer les façons de lutter contre la criminalité, un constat qui apparaîtra évident lorsqu'on aura pris connaissance des budgets présentés ci-dessous.

\section{Où le Canada en est-il aujourd'hui ?}

Il n'est pas surprenant de constater que les mesures plus traditionnellement incorporées au contrôle du crime et à l'application des lois ont continué d'emporter la plus grande part des dépenses gouvernementales, tandis que les initiatives de prévention du crime, telles que celles décrites précédemment, ont dû se débattre et recevoir beaucoup moins d'appui fiscal significatif.

Pendant ses trois premières années d'implantation (1998-2000), le CNPC a reçu 32 millions de dollars per annum pour financer l'ensemble de ses activités (Centre national de prévention du crime, 2008). Il reçoit actuellement 70 millions de dollars par an, soit plus du double de son allocation initiale. À première vue, 70 millions de dollars représentent une somme conséquente, mais comparativement aux sommes engagées dans le financement du système de justice criminelle fédéral et du système de justice pénale dans son ensemble, ce montant est en réalité tout à fait minimal. Ces 70 millions de dollars équivalent à moins de $2 \%$ des dépenses fédérales en matière de justice criminelle ${ }^{21}$. Selon Justice Canada (2003), le Canada a dépensé plus de 13 milliards de dollars par année pour l'ensemble de son système de justice pénale (fédéral, provincial et municipal): par conséquent à peine $0,5 \% \mathrm{du}$

21. Le budget fédéral canadien annuel pour les services de police, le système correctionnel et les tribunaux est évalué à environ 3,6 milliards de dollars. Cela inclut 1,9 milliard de dollars pour les services de police en 2006 (Beattie et Mole, 2007: 11), 1,69 milliard de dollars pour le système correctionnel en 2005/2006 (Comité de la statistique correctionnelle Sécurité publique Canada, 2007: 25), et 90 millions de dollars pour les tribunaux en 2000/2001 (Taylor-Butts, 2002: 7). 
budget total alloué à la justice pénale a été accordé à la prévention du crime.

En outre, ce chiffre de 13 milliards de dollars (qui reflète les données canadiennes de 2003, les plus récentes à avoir été publiées) est probablement une sous-estimation de la somme actuelle, tout comme, au cours des dernières années, l'augmentation bien documentée des dépenses liées aux services de police semble corroborer l'hypothèse d'une tendance globalement à la hausse des dépenses liées à la justice pénale. En 2006, 9,9 milliards de dollars ont été consacrés au maintien de l'ordre (Beattie et Mole, 2007). C'est une augmentation de 4,4\% par rapport à 2005, même après ajustement pour tenir compte de l'inflation, ce qui correspond à un coût de 303 \$ pour chaque Canadien. Les dépenses liées au maintien de l'ordre ont fait l'objet d'un financement en hausse massive ${ }^{22}$, en dépit des recommandations du Comité Horner qui préconisait le contraire (Beattie et Mole, 2007: 10).

En qui concerne le financement des services correctionnels, les données les plus récentes, celles de 2005/2006 publiées dans Aperçu statistique: Le système correctionnel et la mise en liberté (Comité de la statistique correctionnelle Sécurité publique Canada, 2007), ont révélé que trois milliards de dollars sont dépensés au Canada (25). Quant aux données les plus récentes sur le financement des tribunaux, elles proviennent du rapport Dépenses de la justice au Canada (Taylor-Butts, 2002: 7) qui parle d'un budget d'un milliard de dollars au Canada en 2000-2001.

\section{La prévention du crime en Alberta}

Malgré ce portrait décevant, quelques signes montrent que la prévention du crime est de plus en plus reconnue et qu'elle commence à être implantée de façon plus substantielle par les politiciens. La province d'Alberta a récemment créé un groupe de travail nommé The Crime Reduction and Safe Communities Task Force. Au départ, il a été mis sur pied dans le but de recueillir de l'information sur les manières de réduire la criminalité, d'améliorer la sécurité dans la communauté et de développer la confiance du public envers le système de justice pénale

22. La part principale de la hausse des dépenses pour le maintien de l'ordre se situe au palier municipal, avec des contributions totales excédant 5 milliards de dollars, soit 56,6\% du total des dépenses de ce type au Canada en 2006 (Beattie et Mole, 2007; Fédération canadienne des municipalités [FCM], 2008). 
de la province (Alberta, 2008b). Le groupe de travail était composé de neuf membres et présidé par Heather Forsyth. Celle-ci a rencontré des citoyens et acteurs-clés dans 14 communautés de l'Alberta en avril et mai 2007 pour entendre leurs principales inquiétudes et recueillir de l'information sur les meilleures pratiques existantes.

En 2007, l'Institut pour la prévention de la criminalité (IPC, 2007b) de l'Université d'Ottawa a aidé le groupe de travail de l'Alberta à faire la compilation de ces recherches et données afin d'en arriver à une nouvelle stratégie de prévention du crime à l'échelle de la province, incluant une nouvelle vision politique préconisant "Less Law, More Order» (Waller, 2006), soit «moins de répression, plus de sécurité» afin de réduire la victimisation par l'entremise de la prévention. L'IPC a préparé un rapport intitulé Preventive Solutions to Crime in Alberta: from Evidence to Results (IPC, 2007b) qui a regroupé le même type de données probantes que celles décrites dans les deux premières sections de cet article. Le rapport de l'IPC, ainsi que les consultations publiques menées dans les 14 communautés, a incité le groupe de travail de l'Alberta à rédiger son propre rapport intitulé Keeping Communities Safe; Report and Recommendations (Alberta's Crime Reduction and Safe Communities Task Force, 2007) et incorporant la plupart des recommandations-clés de l'IPC quant à l'implantation d'une prévention basée sur des données probantes.

Le gouvernement de l'Alberta a accepté plusieurs de ces recommandations, notamment un plan d'action et un centre de responsabilité qui sont devenus les premières priorités (Alberta, 2008c). Le centre de responsabilité ainsi créé sera dirigé par un sous-ministre adjoint, ce qui le positionne plus haut dans la hiérarchie gouvernementale que ne l'est le CNPC (Alberta, 2007). Le gouvernement d'Alberta devient le premier gouvernement, tous paliers confondus, à consentir à la création d'un centre de responsabilité dirigé par un représentant du pouvoir politique, aussi bien qu'à la mise en place d'un plan d'action de prévention basé sur des données probantes.

Le plan prévoit que 468 millions de dollars seront répartis, sur une période de trois ans, dans une stratégie à trois volets, à savoir : l'application des lois $(25 \%)$, le traitement $(50 \%)$ et la prévention $(25 \%)$ (Alberta, 2008a; 2008c). Dans un communiqué de presse (novembre 2007, voir Waller, 2008), le gouvernement d'Alberta a annoncé l'intention de la province d'agir vigoureusement sur le crime et sur ses causes. Même si l'application des lois continue de faire partie de la 
stratégie proposée, ce programme reste le meilleur exemple que ce pays ait connu en termes d'adoption d'une approche de prévention du crime basée sur des données probantes. En effet, les nouveaux fonds alloués à la prévention équivalent à ceux prévus pour l'application des lois. En outre, Rick Linden, consultant en prévention du crime, professeur à l'Université de Manitoba, et son groupe de recherche Prairie Research Associates, aident présentement l'Alberta à élaborer son plan stratégique et à développer un devis d'évaluation pour l'ensemble de la stratégie. Ils examineront chacun des programmes à implanter et développeront des modèles logiques pour que ces programmes puissent être évaluables.

Les investissements et les efforts multisectoriels de l'Alberta sont les premiers de la sorte au Canada. Ils marquent un progrès, mais ils mettent aussi en évidence le fait que les neuf autres provinces et les deux territoires ne possèdent toujours aucune stratégie significative de prévention du crime, et qu'il n'existe pas de stratégie bien articulée au palier fédéral. Le Québec est une exception partielle dans le portrait d'ensemble, puisqu'on y a adopté une politique ministérielle en prévention de la criminalité (Ministère de la Sécurité publique, 2001), assortie d'un plan d'action et qu'on y a aussi déployé quelques projets de prévention du crime axée sur le risque. Cependant, plusieurs de ces projets de recherche et de développement ont été financés par le CNPC et, à ce jour, la politique provinciale implantée n'inclut pas l'investissement financier recommandé. Au Canada, la prévention du crime reste dominée par les mesures de justice pénales traditionnelles, qui sont pour la plupart réactives et non pas proactives. Jusqu'à présent, au Canada, l'Alberta offre le meilleur exemple d'une prévention efficace du crime qui soit reconnue et implantée de manière significative.

\section{Conclusion}

Cet article a proposé un survol critique des travaux qui traitent de la prévention du crime et de son implantation. Il a d'abord examiné des conclusions provenant de recherches qui présentent des pratiques de prévention considérées efficaces. Les évaluations scientifiques d'initiatives de prévention du crime qui s'attaquent à des facteurs de risque montrent qu'elles réduisent effectivement la criminalité et qu'elles le font avec plus d'efficacité que les pratiques traditionnelles de justice pénale.

La deuxième partie a examiné les travaux, de plus en plus nombreux, qui portent sur des manières de dispenser et d'implanter une prévention 
efficace du crime. Il existe un consensus de plus en plus large entre les experts sur un plan où différents paliers de gouvernement devraient mobiliser des organismes qui peuvent s'attaquer de façon systématique à un ou plusieurs facteurs à partir d'un diagnostic des lacunes dans les services, d'un plan qui vise à les combler, d'un effort concerté pour implanter des programmes corrigeant les failles et enfin d'une évaluation des résultats.

La troisième partie de l'article souligne ce qu'ont fait jusqu'à présent les gouvernements pour implanter une prévention efficace du crime. Sur le plan fédéral, le CNPC dispose de 70 millions de dollars pour un programme de recherche et de développement axé sur les facteurs de risque, mais cette somme représente moins de $2 \%$ des dépenses fédérales en matière de justice criminelle et moins de $0,5 \%$ des dépenses totales en matière de justice pénale au Canada. La seule province où une action préventive basée sur des données probantes a été adoptée est l'Alberta, où a récemment été préparée une stratégie à trois volets axée sur la prévention, l'application des lois et le traitement, incluant de nouveaux budgets pour la prévention qui égalent ceux alloués à l'application des lois.

Cet article pose évidemment la question de savoir ce qui s'est produit en Alberta pour que la prévention du crime puisse y être ainsi mise en valeur. L'utilisation qui a été faite de la perspective «moins de répression, plus de sécurité» (Waller, 2006), puis de l'analyse de l'ICP pourrait suggérer quelques façons de surmonter les résistances bien enracinées par rapport aux pratiques préventives qui, historiquement, ont été observées au Canada. Cela donne un aperçu de ce que d'autres provinces, notamment le Québec, pourraient faire pour aller de l'avant. Car malgré quelques progrès notables, celles-ci ne sont pas encore parvenues à établir à l'échelle provinciale une stratégie basée sur des données probantes.

Premièrement, le groupe de travail de l'Alberta a reçu de l'IPC des recommandations rédigées dans un format facilement accessible. Le contenu de ce rapport évaluait la situation albertaine sur le plan de la criminalité et il décrivait diverses avenues de solutions, mais il le faisait à l'aide d'une terminologie simple. L'IPC a aussi suggéré au groupe de travail de prendre connaissance de Less Law, More Order (Waller, 2006), un livret qui aborde la recherche en matière de prévention efficace du crime dans un langage qui peut être compris par les politiciens et les électeurs. Les recensions systématiques portant sur la prévention efficace, 
citées tout au long de cet article, mettent de l'avant plusieurs preuves de «ce qui fonctionne»; toutefois, elles sont rarement rédigées en des termes profanes et destinées à un auditoire n'appartenant pas aux milieux universitaires.

Deuxièmement, le groupe de travail s'est vu remettre des recommandations claires et pragmatiques. Il est surprenant que, parmi toutes les provinces du Canada, ce soit l'Alberta, une province dirigée par un gouvernement «de droite», qui ait décidé d'investir la somme d'argent la plus importante dans la prévention. Cela peut s'expliquer, au moins en partie, par le fait que les gens ont reçu des recommandations qui étaient pratiques et réalisables, assorties d'objectifs qui correspondaient bien à leurs problèmes de criminalité.

Troisièmement, la vision proposée par Waller (2006) entend déplacer le débat public en criminologie de manière à ce qu'on parle moins du système de justice pénale, des intérêts des contrevenants ou des suspects, et qu'on se centre plus sur les victimes. Le débat public que veut soulever Waller est en grande partie axé sur la manière la plus adéquate de réduire le tort causé aux victimes d'actes criminels. En invitant les victimes d'actes criminels à s'accorder avec les contribuables, il suggère que le système pénal actuel est bien inefficace pour accroître leur sécurité. Au lieu de se concentrer sur les thèmes habituels endossés par les courants dominants du corpus criminologique, tels que le pouvoir des riches et l'impuissance des pauvres, thèse soutenue par la criminologie marxiste, Waller s'en détourne. Il veut poser la question de ce qui peut être fait pour les victimes, plutôt que ce qui peut être fait pour que le système de justice pénale cesse de traiter injustement les pauvres. Les criminologues tels que Reiman (2007) mettent l'accent sur l'idée que le système de justice pénale est un «cycle» qui profite aux individus qui sont en position de pouvoir apporter des changements, et défavorise ceux qui n'ont pas un tel pouvoir. Au lieu de questionner le système et son fonctionnement sous-jacent, Waller (2006) porte son attention sur ce qui risque de faire vraiment une différence dans les taux de crime.

Quatrièmement, la vision avancée par Waller n'endosse pas de recommandations radicales telles l'élimination de la police, du système correctionnel ou des tribunaux. Il ne soutient pas que l'application des peines devrait être retirée de la justice pénale. Au lieu de cela, il se prononce sur la part des budgets massifs de la justice pénale qui devrait être redirigée vers la prévention afin de faire une différence dans la 
réduction du nombre de victimes. Il évite les faux arguments tels que le «dénigrement de la police» ("police bashing») et propose plutôt des recommandations opérantes qui peuvent être facilement intégrées au système en place. C'est probablement grâce à cette approche bien articulée, réaliste et proactive que le gouvernement d'Alberta a été capable d'intégrer plusieurs des solutions fondées sur des données probantes qui lui ont été proposées. La stratégie à trois volets (l'application des lois, la prévention et le traitement) a été adoptée avec vigueur et enthousiasme, lorsqu'on la compare à l'accueil habituellement réservé aux efforts préventifs déployés dans ce pays. Peut-être que le point fort de la stratégie tient au fait qu'elle se range aux côtés des bastions déjà établis de la police, des services correctionnels et des tribunaux plutôt que d'entrer en conflit avec eux. En choisissant d'endosser et de souligner les rôles essentiels que les éléments traditionnels de la justice pénale jouent dans le système, le champ de la prévention du crime pourrait avoir finalement découvert l'insaisissable feuille de route ("elusive roadmap») qui lui assurera une reconnaissance plus continue et une implantation plus conséquente dans le futur.

Cinquièmement, tout ceci a été communiqué au moment où un groupe de travail était à l'œuvre. Au Canada, les commissions d'enquête Archambault, Ouimet et Fauteux ont toutes mené à des réformes significatives de la justice pénale - comme l'ont fait les commissions présidentielles dans les années 1960 aux États-Unis. Bien que toutes les recommandations n'aient pas été implantées, les gouvernements ont investi de nouveaux fonds et adopté de nouvelles lois. Le groupe de travail d'Alberta a utilisé la recherche basée sur des données probantes pour interpréter des sondages d'opinion publique et des préoccupations politiques. Ses 31 recommandations ont été proposées juste avant une élection et elles sont devenues la politique du gouvernement à la suite des élections. Il reste à voir, au cours des prochaines années, dans quelle mesure ces recommandations sont effectivement implantées.

Pour conclure, il se peut que le recadrage des questions de criminologie en débat qui porte sur le sort des victimes soit un des premiers pas pour réduire le crime au moyen de la prévention. Présentement, plusieurs débats en criminologie se sont éloignés d'un intérêt pour la réduction de la victimisation causée par le crime. Un mouvement antiempirisme récent au sein de la discipline a favorisé l'adhésion à des théories radicales et, plus récemment, à des explorations philosophiques sur la «réalité» du monde social. 
La criminologie et ses partisans nous paraissent avoir besoin de retourner à une «réalité» que la majorité des individus perçoit comme étant, somme toute, un bon reflet du monde dans lequel nous vivons. La perspective avancée par Waller représente un pas dans la bonne direction pour la criminologie comme discipline, mais également en tant qu'outil puissant qui pourrait offrir l'opportunité (au public et aux décideurs) d'en arriver à d'importantes réductions des taux de crime et de victimisation.

\section{Références}

Alberta (2007). Government Gets Tough on Crime and its Causes; work to begin immediately on task force recommendations. News Release, 6 novembre.

Alberta (2008a). Alberta's Safe Communities Secretariat Overview. Consulté le 8 novembre 2008, http://justice.gov.ab.ca/safe/default.aspx?id=5407

Alberta (2008b). Crime Reduction and Safe Communities Task Force. Consulté le 8 novembre 2008, http://justice.gov.ab.ca/initiatives/default.aspx?id=5074

Alberta (2008c). Safe Communities Recommendations Action Plan. Consulté le 8 novembre 2008, http://justice.gov.ab.ca/downloads/documentloader. aspx?id $=48560$

Alberta's Crime Reduction and Safe Communities Task Force (2007). Keeping Communities Safe; Report and Recommendations. 27 septembre. Edmonton, Alberta.

Beattie, S., \& Mole, A. (2007). Police Resources in Canada, 2007. Ottawa: Canadian Centre for Justice Statistics. Ministry of Industry. Catalogue no. 85225-XIE.

Begin, P. (1992). Crime and Prevention in Canada. Background Paper. Ottawa: Library of Parliament. Research Branch.

Birkbeck, C. (2005). The Market for Scientific Crime Prevention: A Comparative Study of Canada and Venezuela. European Journal on Criminal Justice Policy and Research. 11, 321-346.

Brantingham, P. Jr. \& Faust F. L. (1976). A Conceptual Model of Crime Prevention. Crime er Delinquency, July, 284-296.

Burrows, M. (2003). Evaluation of the Youth Inclusion Programme: end of phase one report. London: Youth Justice Board.

Conseil Économique et Social des Nations Unies (2002). Guidelines for the Prevention of Crime in Action to Promote Effective Crime Prevention. New York: United Nations, Economic and Social Council, Office for Drug Control and Crime Prevention.

Conseil national de prévention du crime (1996). Building a Safer Canada: A Community-based Crime Prevention Manual. Consulté le 8 novembre 2008, http://ww4.pssp.gc.ca/en/library/publications/general/community/index. html 
Centre national de prévention du crime (2008). Le Centre national de prévention du crime qui relève de Sécurité publique Canada. Consulté le 8 novembre, 2008, http://www.securitepublique.gc.ca/prg/cp/index-fra.aspx

Crooks, C., Wolfe, D., Hughes, R., Jaffe P., \& Chiodo, D. (2008). Development, Evaluation and National Implementation of a School-Based Program to Reduce Violence and Related Risk Behaviour: Lessons from the Fourth R. IPC Review, 2, 109-135.

Cusson, M. (2002). Prévenir la délinquance. Les méthodes efficaces. Paris: Presses universitaires de France.

Cusson, M., Dupont, B. \& Lemieux, F. (2007). Traité de sécurité intérieure. Montréal: $\mathrm{HMH}$.

Farrington, D., \& Welsh, B. (2002). Family-based Crime Prevention. In L. Sherman, D. Farrington, B. Welsh \& D. Mackenzie (eds.), Evidence-Based Crime Prevention (22-55). New York: Routledge.

Farrington, D., \& Welsh, B. (2007). Saving Children from a Life of Crime: Early Risk Factors and Effective Interventions. New York: Oxford University Press.

Fédération canadienne des municipalités (2008). Towards Equity and Efficiency in Policing: A Report on Policing Roles, Responsibilities and Resources in Canada. Consulté le 8 novembre 2008, http://www.fcm.ca//CMFiles/Policing \%20Report1MQG-5292008-4453.pdf

Gottfredson, D. (1997). School-Based Crime Prevention. In S. Lawrence, D. C. Gottfredson, D. Mackenzie, J. Eck, P. Reuter \& S. Bushway (eds.), Preventing Crime: What Works, What Doesn't, What's Promising. Washington: US Department of Justice.

Gottfredson, D., Wilson, D., \& Skroban Najaka, S. (2002). School-Based Crime Prevention. In L. Sherman, D. Farrington, B. Welsh \& D. Mackenzie (eds.), Evidence-Based Crime Prevention (56-164). New York: Routledge.

Greenwood, P. W., Model, K. E., Rydell, C. P., \& Chiesa, J. (1996). Diverting Children from a Life of Crime: Measuring Costs and Benefits. Santa Monica, California: RAND.

Greenwood, P., Karoly, L., Everingham, S., Houbé, J., Killburn, R., Rydell, P., Sanders, M., \& Chiesa, J. (2001). Estimating the Costs and Benefits of Early Childhood Interventions: Nurse Home Visits and the Perry Preschool. In B. Welsh, D. Farrington \& L. Sherman (eds.), Costs and Benefits of Preventing Crime (123-148). Colorado: Westview Press.

Groupe de travail national sur la prévention de la criminalité (2007). Bâtir un Canada plus sûr : Premier rapport du groupe de travail national sur la prévention de la criminalité. Ottawa: Institut pour la prévention de la criminalité.

Hahn, A., Leavitt, T., \& Aaron, P. (1994). Evaluation of the Quantum Opportunities Program. Waltham, Massachusetts: Brandeis University.

Hastings, R. (1996). Crime Prevention and Criminal Justice. In T. O'Reilly Fleming (ed.), Post-Critical Criminology (315-328). Toronto: Prentice Hall.

Horner B. (1993). Twelfth Report of the Standing Committee on Justice and The Solicitor General. House of Commons, February. 
Institut pour la prévention du crime (2007a). IPC Review. Ross Hastings (ed.). Mars. Ottawa.

Institut pour la prévention du crime (2007b). Preventative Solutions to Crime in Alberta: from Evidence to Results. Report prepared for The Crime Reduction and Safe Communities Task Force of Alberta. Ottawa.

Institut pour la prévention du crime $(2008)$. Explanations: Risk Factors Related to Crime. Consulté le 8 novembre 2008, http://www.sciencessociales.uottawa. ca/ipc/eng/why_is_there_so_much_crime.asp

Justice Canada. (2003). JustResearch, 12, 56-57. Consulté le 4 septembre 2007, http://www.justice.gc.ca/en/ps/rs/rep/justresearch/jr12/jr12.pdf

Karoly, L. A., Greenwood, P. W., Everingham, S. S., Hoube, J., Kilburn, M. R., Rydell, C. P., Sanders, M., \& Chiesa, J. (1998). Investing in our children: What we know and don't know about the costs and benefits of early childhood interventions. Santa Monica, CA.: The RAND Corporation.

Landry, L., \& Sinha, M. (2008). Adult Correctional Services in Canada, 2005/2006. Juristat. 28, 6. Consulté le 8 novembre 2008, http://dsp-psd. pwgsc.gc.ca/collection_2008/statcan/85-002-X/85-002-XIE2008006. pdf

Linden, R. (Prairie Research Associates). (2000). Primer on Municipal Crime. Ottawa: Fédération canadienne des municipalités.

Ministère de la Sécurité publique (2001). Politique ministérielle en prévention de la criminalité. Pour des milieux de vie plus sécuritaires. Québec: Ministère de la Sécurité publique.

Olds, D., Henderson, C., Kitzman, H., Eckenrode, J., Cole, R., \& Tatelbaum, R. (1999). Prenatal and Infancy Home Visitation by Nurses: Recent Findings. The Future of Children 9 (1), 44-65.

Organisation mondiale de la santé (2002). Rapport mondial sur la violence et la santé. Genève: Violence and Injuries Prevention.

Organisation mondiale de la santé (2004). Preventing Violence: A Guide to implementing the Recommendations for the World Report on Violence and Health. Genève: Violence and Injuries Prevention.

Pate, A., McPherson, M., \& Silloway, G. (1987). The Minneapolis Community Crime Prevention Experiment: Draft Evaluation Report. Washington: Police Foundation.

Petrosino, A., Turpin-Petrosino, C. \& Finckenauer, J. O. (2000). Well Meaning Programs Can Have Harmful Effects! Lessons From Experiments of Programs Such As Scared Straight. Crime and Delinquency, 46 (3), 354-379.

Comité de la statistique correctionnelle Sécurité publique Canada (2007). Aperçu statistique: Le système correctionnel et la mise en liberté sous condition. Consulté le 8 novembre 2008, http://www.securitepublique.gc.ca/res/cor/ rep/_fl/CCRSO_2007-fra.pdf

Reiman, J. (2007). The Rich Get Richer And the Poor Get Prison. Ideology, class and criminal justice. New York: Allyn \& Bacon.

Rosenbaum, D. (1987). The Theory and Research behind Neighbourhood Watch: Is it a Sound Fear and Crime Reduction Strategy? Crime and Delinquency, 33 (1), 103-134. 
Rosenbaum, D., Lewis, D., \& Grant, J. (1985). The Impact of Community Crime Prevention Programs in Chicago: Can Neighbourbood Organizations Make A Difference? Final Report Vol. 1 to the Ford Foundation. Evanston, IL: Northwestern University, Centre for Urban Affairs and Policy Research.

Rosenbaum, D., Lewis, D., \& Grant, J. (1986). Neighbourhood-Based Crime Prevention: Assessing the Efficacy of Community Organizing in Chicago. In D. Rosenbaum (ed.), Community Crime Prevention: Does it Work? (109-136) Beverly Hills, California: Sage.

Royal Canadian Mounted Police (1949). Law and Order in a Canadian Democracy. Ottawa: Edmond Cloutier.

Sansfaçon, D., \& Welsh, B. (1999). Répertoire-Synthèse II sur la prévention de la criminalité: Analyse comparée d'actions réussies pour la sécurité de la collectivité. Montréal: CIPC.

Schweinhart, L. (2005). The High/Scope Perry Preschool Study Through Age 40: Summary Conclusions and Frequently Asked Questions. Ypsilanti, MI: High/ Scope Press.

Sécurité publique Canada (2008). Plan d'action visant à diminuer efficacement la criminalité. Consulté le 8 novembre 2008, http://www.publicsafety.gc.ca/ prg/cp/_fl/bp-en.pdf

Shaw, M. (2001). The Role of Local Government in Community Safety. Montreal: Centre international de prévention du crime.

Sherman, L. (1997). Family-Based Crime Prevention. In L. Sherman, W. Lawrence, D. Gottfredson, D. Mackenzie, J. Eck, P. Reuter \& S. Bushway (eds,), Preventing Crime: What Works, What Doesn't, What's Promising (4.1-4.42). Washington: US Department of Justice.

Sherman, L., Gottfredson, D., Mackenzie, D., Eck, J., Reuter, P., \& Bushway, S. (1997). Preventing Crime: What Works, What Doesn't, What's Promising. Washington: US Department of Justice.

Sherman L., Farrington, D., Welsh, B., \& Layton Mackenzie, D. (2002). Evidence-Based Crime Prevention. New York: Routledge.

Table ronde sur la prévention de la criminalité (1993). Pour un Québec plus sécuritaire: partenaires en prévention. Rapport de la Table ronde sur la prévention de la criminalité. Québec: Ministère de la Sécurite publique.

Taylor-Butts, A. (2002). Dépenses de la justice au Canada 2000/01. Juristat. 22 , 11. Consulté le 8 novembre 2008, http://www.statcan.gc.ca/pub/85002-x/85-002-x2002011-fra.pdf

Waller, I. (2006). Less Law, More Order: The Truth About Reducing Crime. Westport, CT: Praeger Publishers.

Waller, I. (2008). Reducing Violent Crime requires smart investments in prevention not just Criminal Code amendments. Ottawa: Institut pour la prévention du crime.

Waller, I. \& Weiler, R. (1984). La prévention du crime par le développement social. Ottawa: Conseil canadien de développement social.

Waller, I., Sansfaçon, D., \& Welsh, B. (1999). Crime Prevention Digest II : Comparative Analysis of Successful Community Safety. Montreal: Centre international de prévention du crime. 
Welsh, B., \& Farrington, D. (2002). What works, what doesn't, what's promising, and future directions. In L. Sherman, D. Farrington, B. Welsh \& D. Layton Mackenzie (eds.), Evidence-Based Crime Prevention (405-420). New York: Routledge.

Wolfe, D., Crooks, C., Chiodo, D., Hughes, R., \& Jaffe, P. (2005). Impact of a comprehensive school-based prevention program: changes in adolescents' knowledge, attitudes, and behaviours related to violence, sexual behaviour, and substance use. London ON: CAMH Centre for Prevention Science.

ABSTRACT - This paper gives an overview of the literature on effective crime prevention and its implementation. Scientific evaluations of crime prevention projects that tackle risk factors often reveal that they reduce crime and are often more efficient at doing so than standard criminal justice responses. Inter-governmental organizations agree on the critical steps necessary to mobilise relevant agencies to tackle such risk factors. Despite recommendations by parliamentary committees and a growing number of experts, effective crime prevention has not achieved the prominent role that it could occupy in order to more effectively reduce rates of crime in Canada. However, the recent policy announcement by the province of Alberta may offer some ways in which this resistance might eventually be overcome.

KEYWORDS - Crime prevention, evidence based, program implementation, Canada.

RESUMEN - Este artículo ofrece un panorama de textos científicos relativos a programas de prevención del crimen eficaces y a su puesta en práctica. Las evaluaciones científicas de proyectos de prevención del crimen que abordan los factores de riesgo muestran con frecuencia que pueden reducir la criminalidad y que a menudo son más eficaces que las respuestas del sistema penal. Las organizaciones intergubernamentales coinciden sobre las etapas clave que son necesarias para movilizar a los organismos pertinentes en la lucha contra dichos factores de riesgo. Pese a las recomendaciones de comités parlamentarios y de un número creciente de expertos, la prevención del crimen no desempeña aún el papel preponderante que podría tener a fin de reducir de manera más eficaz la tasa de criminalidad en Canadá. Sin embargo, la reciente política anunciada por la provincia de Alberta sugiere algunas vías a través de las cuales dicha resistencia podría ser superada eventualmente.

PALABRAS CLAVE - Prevención del crimen, prácticas basadas en la evidencia, implantación de programas, Canadá. 